\title{
MOOCEP, un método para construir cursos masivos para adultos mayores: usando una creación MOOCEP
}

\section{(MOOCEP, a Method for Building Massive Online Courses for Elderly People: Using a MOOCEP Creation)}

\author{
Priscila Cedillo", Paola Beltrán1, Paúl Rodriguez-Ch¹, Freddy Serrano², \\ Alexandra Bermeo ${ }^{1}$
}

\begin{abstract}
Resumen:
Hoy en día, la población de adultos mayores se ha incrementado significativamente en relación con el total de la población mundial, así también este segmento en los últimos años ha mostrado una mayor adherencia e interés a los avances tecnológicos, entre ellos la Internet y sus servicios con la finalidad de desarrollar diferentes actividades tales como entretenimiento, información de actualidad, aprendizaje de diferentes tópicos, entre otros. Sin embargo, las aplicaciones y sitios web no consideran los requisitos específicos de las personas de la tercera edad. De ahí, es necesario contar con metodologías, procesos y herramientas, las mismas que tengan en cuenta formas apropiadas de interacción, específicas para este sector vulnerable de la población. Este artículo es una extensión al presentado en la conferencia INCISCOS 2017, en la cual se propuso una metodología para la creación de cursos masivos en línea denominada MOOCEP; $y$ en este trabajo, se ha visto conveniente probar los artefactos creados con ella, por medio de la presentación de dichos recursos a personas de la tercera edad, con la finalidad de realizar una comparativa del antes y después del conocimiento de los diversos temas expuestos en la plataforma de aprendizaje.
\end{abstract}

Palabras clave: MOOC; objetos de aprendizaje; metodología; adulto mayor; andragogía.

\begin{abstract}
:
Today, the population of elderly people has increased significantly in relation to the total world population. In recent years, this segment of population has shown a greater adherence and interest in technological advances, among them the Internet and its services; this, with the aim of develop different activities such as entertainment, obtain current information, learning different topics, among others. However, applications and websites do not take into account the specific requirements of elderly people. Hence, it is necessary to have methodologies, processes and tools that take into account appropriate forms of interaction specific to this vulnerable sector of the population. This article is an extension to the one presented at the INCISCOS 2017 conference, which proposed a methodology for the creation of massive online courses, called MOOCEP. In this work, it has been considered convenient to test the devices created with the use of MOOCEP, by presenting these resources to elderly people, in order to make a comparison of the before and after knowledge of the various topics presented in the learning platform.
\end{abstract}

Keywords: MOOC, learning objects, methodology, elderly people, andragogy.

\footnotetext{
1 Universidad de Cuenca, Cuenca - Ecuador (\{priscila.cedillo, paola.beltran, paul.rodriguezc, alexandra.bermeo\} @ucuenca.edu.ec).

2 Universitat de València, Valencia - España (freddyse@alumni.uv.es).
} 


\section{Introducción}

En las últimas décadas, el campo de la educación, ha experimentado una evolución vertiginosa, apegado a los avances de las Tecnologías de la Información y Comunicación (TIC), así como también un incremento importante del número de usuarios (Arpetti, Baranauskas, \& Leo, 2014). El uso de las TIC en un contexto de aprendizaje es conocido comúnmente como e-Learning. Estos tipos de tecnologías, han introducido muchos desafíos hacia la mejora de la eficiencia de los procesos educativos.

El acceso universal a la tecnología es importante en el campo de la educación y de los sistemas interactivos, debido a la necesidad de promover la igualdad de oportunidades para todos (Gulliksen \& Harker, 2004). De ahí, la efectividad en los procesos de aprendizaje a través de e-learning, consiste en conocer claramente, los objetivos de los usuarios y sus necesidades (Gamage, Femando, \& Perera, 2015). Como resultado de esto, es necesario determinar el público objetivo que utilizará una solución de aprendizaje particular. Adicionalmente, el uso de las TIC por parte de las personas de la tercera edad se ha incrementado significativamente. Las computadoras personales y los dispositivitos tecnológicos están acaparando progresivamente la atención de los adultos mayores con fines de entretenimiento, redes sociales, aprendizaje, salud y telemedicina, entre otros (Tutoky, Babic, \& Wagner, 2013).

Consecuentemente, el objetivo de muchos grupos de investigación es capturar las necesidades de aprendizaje de las personas de la tercera edad cuando utilizan medios electrónicos, con el fin de encontrar los principales requisitos que permitan un aprendizaje efectivo, para incrementar sus habilidades a través de soluciones apropiadas (Tutoky et al., 2013). Asímismo, los cursos masivos abiertos en línea (Massive Open Online CoursesMOOC) son catalogados como "masivos" dado que son diseñados para ser utilizados por miles de estudiantes; "abiertos" porque pueden ser accedidos por cualquier persona; "en línea" porque los usuarios pueden ingresar e interactuar con dichos cursos a través de la Internet y son "cursos" dado que los MOOC tienen una fecha inicial y una final o con un ritmo apegado a la necesidad del aprendiz (Meister, 2013).

El origen de los MOOC se remonta al año 2008, pero no es sino hasta el año 2012, cuando el New York Times declara a este, el año de los MOOC, debido principalmente a que las principales plataformas tales como Coursera, Udacity o edX ganaron muchos adeptos.

De acuerdo con Bong \& Chen (2016), casi un 10\% de estudiantes de MOOC son adultos mayores; algunos cursos de plataformas tales como Coursera fueron analizados y se concluyó que estos recursos no reúnen los criterios de accesibilidad necesarios para personas de la tercera edad. Además, no están alineados con las guías de contenido presentadas en WCAG 2.0 (Web Accessibility Guidelines) (W3C, 2017). Por tanto, dentro de lo que conocemos, no existen investigaciones orientadas a apoyar la creación de MOOC para personas de la tercera edad basadas en un método específico. De ahí, en el congreso INCISCOS 2017, se presentó un método para la creación de MOOC orientados a la tercera edad denominado MOOCEP (Beltrán, Cedillo, Rodríguez-Ch, \& Bermeo, 2017); sin embargo, en ese estudio no se llevaron a cabo pruebas de dichos recursos, que valoren la efectividad de los MOOC construidos mediante MOOCEP. En consecuencia, este artículo contribuye con una extensión a Beltrán et al. (2017) realizada en una muestra de 36 adultos mayores, en la cual se ha realizado un test inicial y otro final que permite concluir que los MOOC construidos con MOOCEP permiten mejorar significativamente el conocimiento sobre el tema que se necesita trasmitir al adulto mayor.

La estructura de este artículo es la siguiente: la sección 2 presenta trabajo relacionado que permite conocer las contribuciones en este campo de investigación. Luego, la sección 3, ofrece el método propuesto para la creación de MOOC para personas de la tercera edad, la sección 4 se refiere a la evaluación realizada en cuanto al aprendizaje de formas correctas de alimentación por parte de los adultos mayores. Finalmente, en la sección 5 son expuestas las conclusiones y las líneas de investigación futuras. 


\section{Trabajo relacionado}

Se analizó trabajo relacionado tomando en consideración algunas revisiones sobre MOOC. En dichos estudios, se observó si los MOOC reunían ciertas características para mejorar la experiencia de las personas de la tercera edad, tales como, técnicas, estrategias y criterios de accesibilidad orientados a mejorar su aprendizaje. De ahí, se lograron abstraer trabajos tales como (Al-Mouh, Al-Khalifa, \& Al-Khalifa, 2014; Sánchez-Gordón \& Luján-Mora, 2013; UTEID, 2014). En donde, Al-Mouh et al. (2014), realizó pruebas de accesibilidad en cursos desplegados en la plataforma Coursera; sin embargo, los MOOC ahí analizados, no reunían los criterios mínimos de accesibilidad para personas de la tercera edad. Además, Sánchez-Gordón \& Luján-Mora (2013) toman en cuenta algunas consideraciones para la creación de MOOC orientados a adultos mayores. Estos autores analizan algunos cursos desplegados en Coursera para ejecutar la evaluación de los criterios de accesibilidad, basados en las guías Web Content Accesibility Guidelines (WCAG 2.0) y la iniciativa de accesibilidad Web (Web Accesibility Initiative: Ageing Eduaction and Harmonization WAI-AGE), estudios en los cuales se han encontrado problemas de accesibilidad para adultos mayores, los mismos que necesitan ser abordados.

Asímismo, Barajas Saavedra (2007), presenta un modelo conceptual para la creación de objetos de aprendizaje (Learning Objects-LO). Este modelo está basado en el uso de cualquiera de los siguientes dos métodos de la Ingeniería del Software: i) ciclos de prototipado evolutivo, ii) programación extrema. Luego, ellos proponen una metodología, la misma que tiene cuatro fases: a) análisis, b) diseño, c) desarrollo, y d) evaluación. No obstante, esta metodología no está orientada a crear artefactos para adultos mayores. La Unidad para la Educación Tecnológica e Innovación en el Aprendizaje (UTEID, 2014), estableció una guía metodológica para el pleamiento, diseño y entrega de MOOC, esta también contiene guías para la creación de contenidos audiovisuales, mecanismos de evaluación, herramientas de comunicación y soporte durante el diseño y creación de un MOOC, ellos tampoco establecieron pautas y guías orientadas a MOOC para personas de la tercera edad. Así también, la metodología de Sanz, Moralejo, \& Barranquero (2017) está basada en cinco fases (análisis, diseño, desarrollo, publicación y evaluación). Esta metodología está centrada en: i) incrementar el nivel de detalle para alcanzar el estado de desarrollo, ii) guiar a los profesores a la construcción de objetos de aprendizaje, y iii) diseñar guías para integrar técnicas pedagógicas y tecnológicas. Al igual que otras muchas soluciones, esta metodología no está orientada a personas de la tercera edad. Finalmente, Baruque \& Melo (2004) proponen una metodología llamada Instructional System Development (ISD), la cual está basada en objetos de aprendizaje, orientada al diseño y desarrollo de contenido educacional. Este se fundamenta en el modelo instruccional de Análisis, Diseño, Desarrollo, Implementación y Evaluación (ADDIE) (Ghirardini, 2011); su público objetivo es el público en general.

Como se ha mostrado en los estudios relacionados, poca atención se ha prestado a la creación de metodologías que permitan la construcción de MOOC orientados a personas de la tercera edad, al tener en cuenta sus necesidades y preferencias.

\section{El Método Massive Open Online Courses for Elderly People (MOOCEP)}

Para crear MOOC orientados a las personas de la tercera edad, es necesario establecer una guía que permita seguir los pasos necesarios hacia la construcción de este tipo de artefactos. De ahí, el Massive Open Online Courses for Elderly People (MOOCEP) se ha creado al considerar este objetivo. Esta guía considera la inclusión de técnicas andragógicas y estrategias necesarias para la construcción de MOOC que impulsa el aprendizaje de personas de la tercera edad, orientado a diversos tópicos que contribuyan a la mejora de su estilo de vida. Este método presenta una solución flexible y extensible, 
adaptable a cualquier temática; para diseñar y crear estos artefactos, se toman en consideración procesos de aprendizaje que puedan variar considerablemente tanto en complejidad como en tamaño como el presentado por Ghirardini (2011), con el cual MOOCEP está alineado.

En esta sección se explica paso a paso el método MOOCEP, con una breve explicación de cada una de sus actividades. Cada actividad está presentada en la Figura 1, con los artefactos y guías que la componen; además, la figura presenta una numeración, la misma que permite ilustrar claramente cada uno de los pasos a seguir.

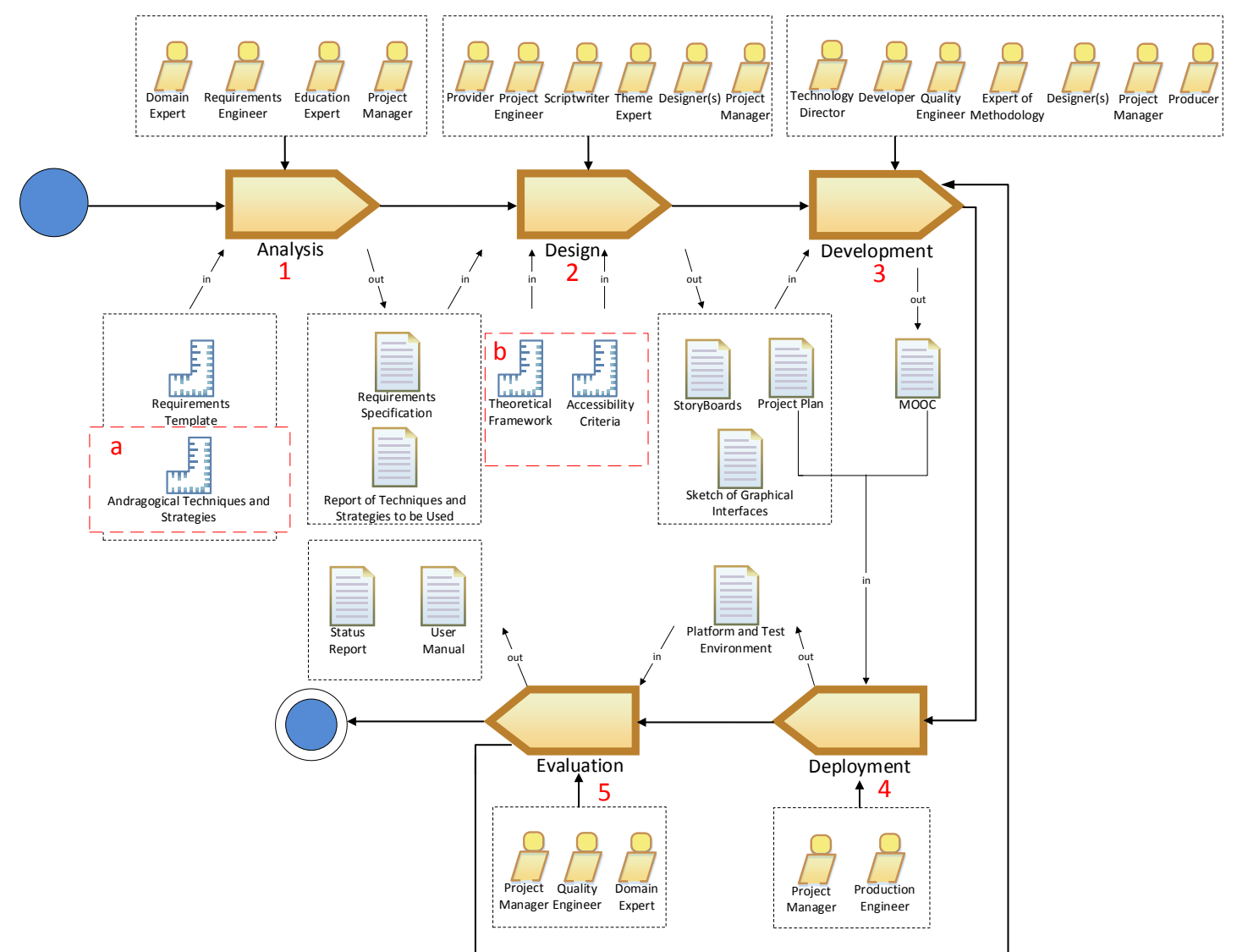

Figura 1. Vista general del método MOOCEP (Beltrán et al., 2017)

Dentro de MOOCEP es importante tener en consideración que este método incluye dos ventajas significativas con respecto a otros métodos de carácter general, a) la actividad de análisis contiene técnicas andragógicas y estrategias, lo cual permite un mejor entendimiento de los MOOC cuando son usados por sus stakeholders (ver Figura 1-a); y b) la actividad de diseño considera criterios de accesibilidad (Arch, 2009; W3C, 2017) para mejorar la interacción humana con la solución; estos criterios consideran problemas de salud y restricciones propios de la edad (ver Figura 1-b).

A continuación, se describe cada una de las actividades más detalladamente:

Análisis: en esta actividad (ver Figura 1-1), el MOOC debe ser definido de la siguiente manera: i) las metas del curso y las necesidades que guían la creación del MOOC, las cuales son proporcionadas por el experto del dominio, con la ayuda del ingeniero de requisitos y el administrador del proyecto; ii) el análisis del público objetivo, el cual define la gente a la cual el MOOC estará orientado, este análisis es hecho por el administrador del proyecto y el experto de dominio; iii) la identificación y clasificación del contenido del curso. Esta área es hecha por el experto del dominio, el administrador del proyecto y el ingeniero de requisitos. Y, finalmente iv) la selección de las técnicas andragógicas y estrategias que soportan el proceso de aprendizaje. El experto en 
educación está a cargo de esta tarea. Una descripción completa de esta actividad ha sido presentada en (Rodríguez-ch, Cedillo, Beltrán, \& Ortiz, 2017).

Diseño: esta actividad es mostrada en la Figura 1-2. Aquí, i) la planeación del proyecto es determinada mediante los requisitos y el reporte de técnicas y estrategias andragógicas provistas por la fase de análisis. Esta tarea es ejecutada por el ingeniero de proyectos, el administrador del proyecto y el proveedor, quien ofrece los servicios a ser usados durante el desarrollo del MOOC (proveedor de hosting, estudios de grabación, servicios de impresión). Los storyboards son definidos por el escritor del guión quien los escribe con la ayuda del experto en el tema y iii) el sketch de la interfaz gráfica es ejecutada por los diseñadores. La principal diferencia del análisis en este método versus los tradicionales, es el uso de criterios de accesibilidad orientados a las personas de la tercera edad y las consideraciones obtenidas por los requisitos andragógicos establecidos en la fase de análisis.

Desarrollo: esta labor es mostrada en la Figura 1-3. El principal objetivo de esta tarea es la implementación del MOOC. El contenido puede cambiar según los storyboards, interactividad y recursos multimedia provistos por la actividad de diseño. Esta actividad contiene: i) el desarrollo de contenido y multimedia (textos, imágenes, audios, interacciones, animaciones, evaluaciones y pruebas), las cuales son seleccionadas de acuerdo con el plan del proyecto los storyboards y los sketches. Este proceso de desarrollo puede ser guiado utilizando cualquier método de desarrollo de software (p. ej. Scrum, XP, cascada).

Despliegue: esta tarea es ilustrada en la Figura 1-4. Aquí, el MOOC es cargado sobre un servidor y desplegado para ser accedido por los adultos mayores. En esta actividad, el jefe del proyecto coordina la entrega del MOOC y el ingeniero de producción prepara la plataforma para que el MOOC sea desplegado.

Evaluación: la última actividad es mostrada en la Figura 1-5, esta corresponde a la fase de evaluación de la efectividad cuando el MOOC es usado por las personas de la tercera edad. En esta fase se pueden emplear métodos de evaluación tanto cualitativos como cuantitativos utilizados para probar artefactos creados dentro de la Ingeniería del Software.

Con respecto a los artefactos y guías que intervienen en la metodología (fase de análisis y diseño), se puede encontrar una explicación detallada de cada uno de ellos en: Beltrán et al. (2017); Cedillo et al. (2017), Rodríguez-ch et al. (2017). Además, en trabajos futuros, se plantea abordar por completo todas las actividades y los elementos involucrados.

Finalmente, en la siguiente sección, se muestra una evaluación efectuada de uno de los productos construidos a partir de esta metodología.

\section{Evaluación de la metodología MOOCEP}

En esta sección se presentan los resultados de la evaluación de un MOOC construido con sujeción a la metodología MOOCEP. Aquí, se tiene en cuenta el tema de alimentación del adulto mayor, en el cual un grupo de médicos ha aportado con formas adecuadas de alimentación para adultos mayores; además, se ha indicado al adulto mayor, que antes de realizar cualquier régimen de alimentación específica, consulte a su nutricionista y profesional de la salud.

Para ello y de acuerdo con el paradigma Goal-Question-Metric (GQM) (Basili \& Rombach, 1988), se ha planificado el objetivo de la evaluación de la siguiente manera: Se evaluará el aprendizaje del adulto mayor, cuando este hace uso de un MOOC construido a base de la metodología MOOCEP; con el propósito de: evaluar la eficiencia de MOOCEP con respecto al conocimiento previo del adulto mayor; desde el punto de vista de: los usuarios del MOOC, en este caso las personas de la tercera edad; en el contexto de: adultos mayores de tres instituciones que promueven el bienestar de dichas personas en la ciudad de Cuenca, Ecuador. 
La pregunta de investigación es: ¿El MOOC construido según la metodología MOOCEP ha ayudado al adulto mayor a adquirir conocimientos acerca de buenos hábitos de alimentación?

Esta pregunta de investigación ha sido evaluada a través de la prueba de las siguientes hipótesis:

$\mathrm{H} 1_{0}=$ Los adultos mayores empeoraron su conocimiento sobre alimentación, al utilizar el MOOC producido con MOOCEP.

$\mathrm{H} 2_{0}=$ Los adultos mayores mejoraron su conocimiento sobre alimentación, al hacer uso del MOOC producido con MOOCEP.

\section{Planeación de la evaluación}

El contexto de la evaluación está determinado por un producto construido con sujeción a la metodología MOOCEP, la selección del MOOC que servirá para evaluar el conocimiento del adulto mayor y la selección de los participantes.

Con respecto al ejercicio a ser aplicado, será utilizado un MOOC de alimentación, el mismo que fue construido según la metodología MOOCEP propuesta por Beltrán et al. (2017) y que será utilizado por 36 adultos mayores de la ciudad de Cuenca, Ecuador. Cabe destacar que esta evaluación no constituye un proceso experimental como tal, dada la no aleatorización de los sujetos que intervienen, aun cuando en esta ocasión sí se ha desarrollado el ejercicio en un ambiente controlado.

\section{Tareas a ser realizadas}

Se inició con una breve prueba inicial de 11 preguntas, que sirvió como línea base para la evaluación presentada, acto seguido se instruyó al adulto mayor en cómo utilizar el MOOC, dándole asistencia a fin que pueda visualizar los recursos educativos. Cabe indicar que se obtuvieron impresiones acerca de la dificultad del uso de la plataforma en la cual el MOOC fue desplegado. Se utilizó una plataforma específica para MOOC provista por el Consorcio Ecuatoriano para Internet Avanzado. El recurso puede ser accedido en: http://educacionvirtual.cedia.org.ec/. Luego de revisado el MOOC, el adulto mayor respondió a las 11 preguntas nuevamente a fin de descubrir si la información del MOOC fue transmitida de manera satisfactoria.

\section{Operación y ejecución}

Para la ejecución de la evaluación se tomaron en cuenta los resultados del test inicial y el test final, en los cuales se obtuvieron los siguientes resultados, a través del procesamiento de la información mediante el programa informático SPSS v22. La duración del ejercicio fue de alrededor de 1 hora; la misma que incluyó el test inicial o pretest, el test final o posttest y la reproducción y seguimiento del MOOC. Los resultados de los estadísticos descriptivos se muestran en la Tabla 1.

Tabla 1. Estadísticos descriptivos pre y post test aplicados al adulto mayor

\begin{tabular}{lcccc}
\hline Evaluación & Mínimo & Máximo & Media & Desviación Estándar \\
\hline Pretest & 0 & 7 & 3,6667 & 1,87 \\
Postest & 1 & 9 & 4,9167 & 2,32 \\
\hline
\end{tabular}

La distribución de los datos, no presentó un comportamiento normal en el pretest, se utilizó Shapiro Wilk para muestras menores a 50 sujetos (ver Tabla 2), fue necesario utilizar el test de Wilcoxon para muestras no paramétricas relacionadas, a fin de lograr la obtención de la significancia. Para determinar la significancia se tomaron en cuenta los umbrales presentados en la Tabla 3. 
Tabla 2. Test de normalidad Shapiro-Wilk

\begin{tabular}{lccc}
\hline Evaluación & Estadístico & Sujetos & Sig. \\
\hline Pretest & 0.939 & 36 & 0.047 \\
Postest & 0.958 & 36 & 0.184 \\
\hline
\end{tabular}

Tabla 3. Niveles de significancia (Moody, 2001)

\begin{tabular}{ll}
\hline Valor de Significancia & Rango \\
\hline No significativo & $\mathrm{p}>0.1$ \\
Baja significancia & $\mathrm{p}<0.1$ \\
Media significancia & $\mathrm{p}<0.05$ \\
Alta significancia & $\mathrm{p}<0.01$ \\
Muy alta significancia & $\mathrm{p}<0.001$ \\
\hline
\end{tabular}

En la Figura 2 se presentan los diagramas de caja que ilustran el pre y el postest.

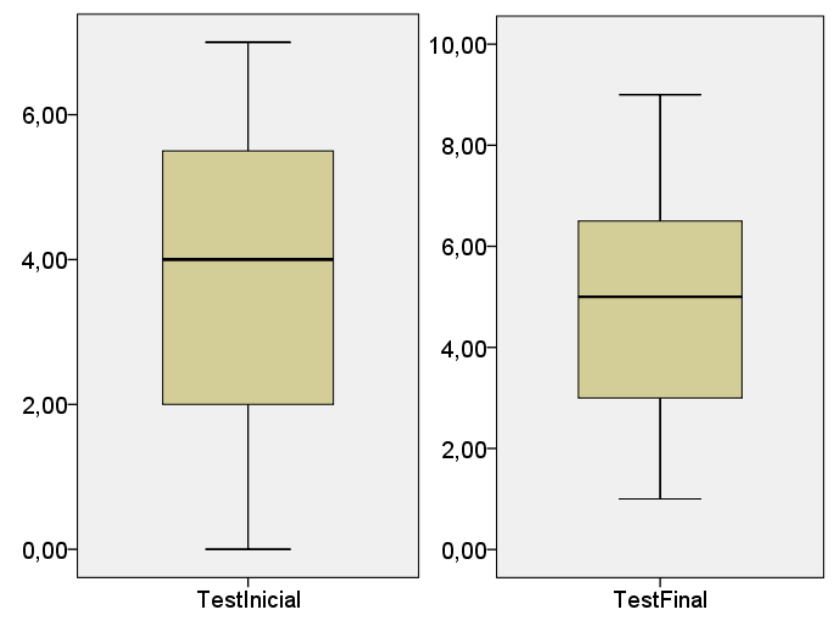

Figura 2. Diagramas de caja para el pre y el postest

Finalmente, luego del procesamiento del pretest y el postest se encontró una alta significancia (0.002) con relación al aprendizaje sobre alimentación del adulto mayor como se muestra en la Tabla 4, valores resultantes de la aplicación de la prueba de Wilcoxon.

Tabla 4. Estadísticos de prueba

\begin{tabular}{lc}
\hline & Estadístico \\
\hline$Z$ & -3.034 \\
Sig. asintótica & $\mathbf{0 . 0 0 2}$ \\
\hline
\end{tabular}

\section{Discusión de los resultados de la evaluación}

Dentro de la evaluación se visitaron varios centros lúdicos y lugares en donde los adultos mayores suelen reunirse a fin de socializar, ahí se efectuaron los ejercicios y los test producto de este estudio.

Al momento de realizar las evaluaciones, se pudo notar de una manera acentuada que no es lo mismo utilizar los MOOC con adultos mayores de edad avanzada versus aquellos que están recién formando parte de este segmento. De ahí, es necesario, utilizar diferentes técnicas andragógicas según la edad del sujeto y del público objetivo. Asímismo, y con gran satisfacción se puede observar que el desarrollar este tipo de ejercicios con los adultos mayores, constituye para ellos una sensación de bienestar e integración, según se pudo constatar en cada uno de los lugares visitados. Cabe destacar que este estudio no 
fue interventivo, sino únicamente se proporcionó información al adulto mayor en cuanto a la pirámide alimenticia y a los alimentos disponibles y saludables según cada región y las mejores formas de alimentarse, indicándoles claramente que antes de adoptar cualquier dieta o programa de nutrición deben consultar a su médico de confianza o nutricionista; por lo cual no hizo falta acudir a comités de ética.

Finalmente, los resultados mostraron claramente la adopción efectiva de conocimiento sobre la materia que trató el MOOC, con resultados significativos con respecto al conocimiento previo adquirido a lo largo de su vida de manera tradicional por parte del adulto mayor.

\section{Conclusiones y recomendaciones}

En este estudio se presentó una evaluación del método MOOCEP, el mismo que consta de 5 fases que permiten la creación de MOOC orientados a personas de la tercera edad. Este artículo muestra resultados prometedores en esta área que nos proporcionan luces acerca de maneras de perfeccionar el método para lograr la mejora del conocimiento y aprendizaje de adultos mayores. Como parte de este método, se muestra la adopción de técnicas propicias para lograr el entendimiento de las diferentes temáticas a transmitir a través de los MOOC; así como también, la adopción de funciones de accesibilidad en este tipo de soluciones. De este modo, se proponen formas para mejorar la situación del adulto mayor en cuanto al aprendizaje, con soluciones a sectores vulnerables que muchas veces se ven relegados y para los cuales no se tienen en consideración herramientas tecnológicas propicias y adecuadas a su edad y limitaciones propias del envejecimiento.

Este trabajo constituye una extensión al trabajo presentado en la conferencia INCISCOS 2017. Y se planea en trabajos futuros aplicar pruebas sobre las percepciones del adulto mayor en cuanto a los MOOC creados para ellos, así como también realizar evaluaciones con los actores de la metodología a fin que esta sea de más fácil aplicación.

\section{Agradecimientos}

Se agradece a CEDIA por el soporte brindado durante la creación y despliegue de la solución, así como también a los fondos provistos en la convocatoria CEPRA-X con los cuales se hizo posible la realización de estos estudios. Además, se agradece a la Universidad Católica de Cuenca por el soporte en la creación de los MOOC, también a la Facultad de Medicina de la Universidad de Cuenca, quienes dieron sus sugerencias sobre el contenido del MOOC de alimentación, utilizado para probar el método aquí presentado.

\section{Bibliografía}

Al-Mouh, N. A., Al-Khalifa, A. S., \& Al-Khalifa, H. S. (2014). A First Look into MOOCs Accessibility. In K. Miesenberger, D. Fels, D. Archambault, P. Pe\$lbackslashl\$vnáz, \& W. Zagler (Eds.), Computers Helping People with Special Needs: 14th International Conference, ICCHP 2014, Paris, France, July 9-11, 2014, Proceedings, Part I (pp. 145-152). Cham: Springer International Publishing. https://doi.org/10.1007/978-3319-08596-8_22

Arch, A. (2009). WAI Guidelines and Older Web Users: Findings from a Literature Review. Arpetti, A., Baranauskas, M. C. C., \& Leo, T. (2014). Eliciting Requirements for Learning Design Tools. In C. Rensing, S. de Freitas, T. Ley, \& P. J. Muñoz-Merino (Eds.), Open Learning and Teaching in Educational Communities: 9th European Conference on Technology Enhanced Learning, EC-TEL 2014, Graz, Austria, September 16-19, 2014, Proceedings (pp. 1-14). Cham: Springer International Publishing. https://doi.org/10.1007/978-3-319-11200-8_1

Barajas Saavedra, A. (2007). Modelo Instruccional para el Diseño de Objetos de Aprendizaje: Modelo MIDOA. E-Spacio.Uned.Es, 52(449), 0. Retrieved from http://e- 
spacio.uned.es/fez/eserv.php?pid=bibliuned:19245\&dsID=n03barajas07.pdf

Baruque, L. B., \& Melo, R. N. (2004). Learning theory and instruction design using learning objects. Journal of Educational Multimedia and Hypermedia, 13(4), 343-370.

Basili, V. R. (1992). The Experimental Paradigm in Software Engineering. In Workshop on Experimental Software Engineering Issues: Critical Assessment and Future Directions (pp. 1-12). London, UK: LNCS 706. https://doi.org/10.1007/3-540-57092-6_91

Basili, V. R., \& Rombach, H. D. (1988). The TAME Project: Towards Improvement-Oriented Software Environments. IEEE Transactions on Software Engineering, 14(6), 758-773. https://doi.org/10.1109/32.6156

Beltrán, P., Cedillo, P., Rodríguez-Ch, P., \& Bermeo, A. (2017). MOOCEP: Towards a Method for Building Massive Open Online Courses for Elderly People. In International Conference on Information Systems and Computer Science. Quito, Ecuador.

Bong, W. K., \& Chen, W. (2016). How Accessible Are MOOCs to the Elderly? In K. Miesenberger, C. Bühler, \& P. Penaz (Eds.), Computers Helping People with Special Needs: 15th International Conference, ICCHP 2016, Linz, Austria, July 13-15, 2016, Proceedings, Part I (pp. 437-444). Cham: Springer International Publishing. https://doi.org/10.1007/978-3-319-41264-1_60

Cedillo, P., Beltrán, P., \& Rodríguez-ch, P. (2017). Evaluation of the Accessibility of MOOCs Oriented to Elderly People Evaluación de la Accesibilidad de MOOC Orientados a la Tercera Edad. Maskana, (Special Issue), 321-332.

Gamage, D., Femando, S., \& Perera, I. (2015). Effectiveness of eleaming: Grounded theory approach. In Moratuwa Engineering Research Conference (MERCon), 2015 (pp. 336341). https://doi.org/10.1109/MERCon.2015.7112369

Ghirardini, B. (2011). E-learning methodologies: A guide for designing and developing elearning courses. Food and Agriculture Organization of the United Nations (FAO). https://doi.org/l2516E/1/11.11

Gulliksen, J., \& Harker, S. (2004). The software accessibility of human-computer interfaces--ISO Technical Specification 16071. Universal Access in the Information Society, 3(1), 6-16. https://doi.org/10.1007/s10209-003-0079-1

Meister, J. (2013). How MOOCs Will Revolutionize Corporate Learning and Development.

Moody, D. L. (2001). A Practical Method for Representing Large Entity Relationship Models, P.h.D. Thesis. University of Melbourne, Australia.

Rodríguez-ch, P., Cedillo, P., Beltrán, P., \& Ortiz, J. (2017). MOOCEP : A Method for Building Massive Open Online Courses for Elderly People The Analysis Activity. In 47th Annual Frontiers in Education (FIE). Indianápolis, USA.

Sánchez-Gordón, S., \& Luján-Mora, S. (2013). Web accessibility of MOOCs for elderly students. In Information Technology Based Higher Education and Training (ITHET), 2013 International Conference on (pp. 1-6). https://doi.org/10.1109/ITHET.2013.6671024

Sanz, C., Moralejo, L., \& Barranquero, F. (2017). Metodología CROA. Retrieved February 11, 2018, from http://croa.info.unlp.edu.ar/

Tutoky, G., Babic, F., \& Wagner, J. (2013). ICT-based solution for elderly people. In Emerging eLearning Technologies and Applications (ICETA), 2013 IEEE 11th International Conference on (pp. 399-404). https://doi.org/10.1109/ICETA.2013.6674466

UTEID. (2014). Guía Metodológica para la Planificación, Diseño e Impartición de MOOC. Madrid, Spain.

W3C. (2017). Web Content Accessibility Guidelines (WCAG) Overview. Retrieved April 15, 2017, from https://www.w3.org/WAl/intro/wcag 